\title{
Acrodysostosis associated with hypercalcemia
}

\author{
Mehmet Kirnap, ${ }^{1}$ Mustafa Calis, ${ }^{1}$ Cumali Gokce, ${ }^{2}$ Selim Kurtoglu, ${ }^{3}$ Mustafa Ozturk, ${ }^{4}$ \\ Fahrettin Kelestimur ${ }^{5}$
}

Departments of ${ }^{1}$ Physical Medicine and Rehabilitation, ${ }^{3}$ Pediatric Endocrinology, ${ }^{4}$ Radiology, ${ }^{5}$ Endocrinology, Erciyes University Medical School, Kayseri, ${ }^{2}$ Department of Endocrinology, Mustafa Kemal University Medical School, Hatay, Turkey

\begin{abstract}
An 18-year-old man was admitted to the clinic complaining of deterioration in the function of his hands and feet. The clinical examination revealed that his movements were clumsy and that he had disproportionally short limbs. In addition, he also had facial abnormalities of frontal bossing, hypertelorism, maxillary hypoplasia, broad low nasal bridge, short upturned nose with anteverted nostrils and triangular mouth. All extremities appeared short with stubby fingers and toes and with broad hands and wrinkling of the dorsal skin. Chromosomal analysis showed a normal $(46, \mathrm{XY})$ karyotype. $\mathrm{X}$-ray studies revealed broad, short metacarpals and phalanges with cone-shaped epiphyses and brachycdactyly and a diagnosis of peripheral dysostosis was confirmed by the characteristic radiographic appearance of the hands. Serum calcium and alkaline phosphatase levels were high, parathormone (PTH) was low, but 25 (OH) Vitamin $D$, albumin, and 24 hour urine calcium levels were in the normal range. Based on these findings, a diagnosis of acrodysostosis associated with hypercalcemia was made. To the best of our knowledge, this represents the first description of this syndrome.
\end{abstract}

Key words: Acrodysostosis, Albright's hereditary osteodystrophy, Hypercalcemia

\section{INTRODUCTION}

Acrodysostosis is a rare syndrome characterized by peripheral dysostosis, nasal hypoplasia, mental retardation and other skeletal abnormalities. ${ }^{1-3}$ Although the mode of inheritance is thought to be autosomal dominant, most cases of acrodysostosis appear to be sporadic. ${ }^{4,5}$ To date, about 50 cases have been reported in the international literature and the cause still remains obscure. It should be noted here that Albright's

Address for correspondence:

Prof. Dr. Fahrettin Kelestimur, Department of Endocrinology, Erciyes University Medical School, 38039, Kayseri, Turkey, Fax: +90 35243758 07, e-mail:cumaligokce@yahoo.com Received 31-01-2013, Accepted 05-06-2013 hereditary osteodystrophy ( $\mathrm{AHO})$ has the same clinical features, but molecular, radiological and biochemical studies suggest that acrodysostosis is clinically different from pseudo-hypoparathyroidism. ${ }^{6-8}$ Patients with AHO may have hypocalcaemia or normocalcemia known as pseudo - pseudo - hypoparathyroidism. ${ }^{9}$ Here we describe a patient with acrodysostosis associated with hypercalcemia. According to our knowledge, it is the first case of acrodysostosis with hypercalcemia reported in the literature.

\section{CASE PRESENTATION}

An 18-year-old man was admitted to the clinic complaining of deterioration in the function of his 
hands and feet, while examination revealed that his movements were clumsy. There was no arthritis in any of the patient's joints. He had disproportionally short limbs and facial abnormalities of frontal bossing, hypertelorism, maxillary hypoplasia, broad low nasal bridge, short upturned nose with anteverted nostrils and triangular mouth. There was no hearing abnormality. All extremities appeared short with stubby fingers and toes and with broad hands and wrinkling of the dorsal skin. Figure 1 depicts the facial abnormalities showing hypertelorism, maxillary hypoplasia, broad low nasal bridge, short upturned nose with anteverted nostrils and triangular mouth. Figure 2 depicts the hands appearing short with stubby fingers and $\mathrm{x}$ ray studies revealing broad, short metacarpals and phalanges with cone-shaped epiphyses and brachycdactyly. Thus, the diagnosis of peripheral dysostosis was confirmed by the characteristic radiographic appearance of the hands. No skeletal abnormalities were observed in siblings or relatives and the parents were normal. Neck ultrasonography and tomography

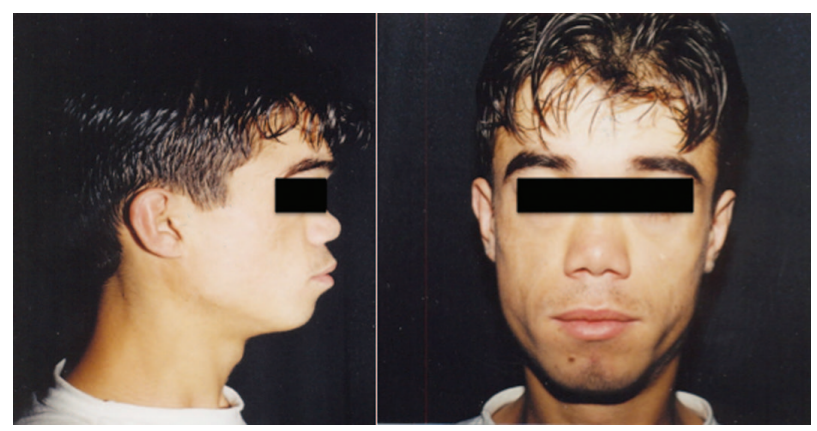

Figure 1. Facial abnormalities showing hypertelorism, maxillary hypoplasia, broad low nasal bridge, short upturned nose with anteverted nostrils and triangular mouth.

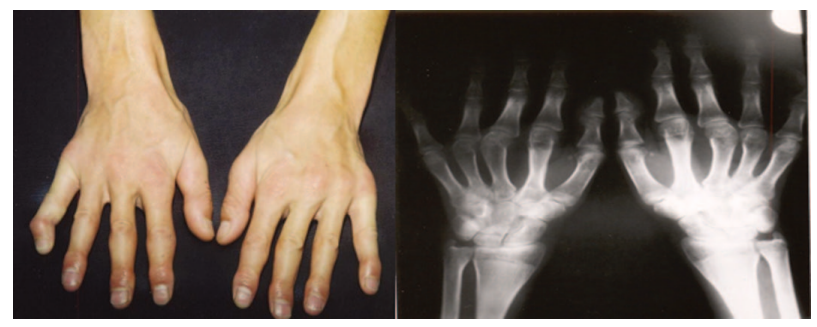

Figure 2. Hands appearing short with stubby fingers and x-ray studies revealing broad, short metacarpals and phalanges with cone-shaped epiphyses and brachycdactyly. were normal. A standard intelligence quotient (IQ) test produced a normal score.

Routine biochemical and laboratory studies are depicted in Table 1. Serum calcium and alkaline phosphatase levels were high, parathormone (PTH) was low, but $25(\mathrm{OH})$ Vitamin $\mathrm{D}$, albumin and 24 hour urine calcium levels were in the normal range. No abnormality of urinary excretion of mucopolysaccharides was detected. Chromosomal analysis showed a normal (46, XY) karyotype.

Table 1: Biochemical Parameters

\begin{tabular}{lcc}
\hline & Patient & $\begin{array}{c}\text { Normal } \\
\text { range }\end{array}$ \\
\hline Calcium $(\mathrm{mg} / \mathrm{dl})$ & $11.2,10.6,11.4,11.1$ & $8.5-10$ \\
Phosphate $(\mathrm{mg} / \mathrm{dl})$ & $3.6,3.8,3.4,3.4$ & $2.5-4.8$ \\
Alkaline phosphatase $(\mathrm{U} / \mathrm{L})$ & $308,298,312,325$, & $100-280$ \\
Total protein gr/dl & 7.5 & $6 .-8.3$ \\
Albumin gr/dl & 4.9 & $3.5-5$ \\
iPTH (pg/ml) & $26,17,5$ & $13-66$ \\
25-OH Vitamin D (ng/ml) & 17 & $10-40$ \\
Ca (24 hour urine) $\mathrm{mg} / \mathrm{day}$ & 250,275 & $100-300$ \\
\hline
\end{tabular}

\section{DISCUSSION}

Acrodysostosis is characterized by short hands with peripheral dysostosis, a flat nasal bridge, skeletal abnormalities of the mandible, skull and spine and mental deficiency. ${ }^{1-3}$ This disorder may be associated with spinal canal stenosis, epiphyseal stippling, Gaucher disease and other disorders. ${ }^{4,10-13}$ Hypercalcemia in a patient with acrodysostosis has not, as far as we know, been reported previously. Shortening of the tubular bones of the hands and feet with cone-shaped epiphyses is defined as peripheral dysostosis and is common to many syndromes including acrodysostosis and AHO or pseudohypoparathyroidism. ${ }^{6,7}$ In both disorders, clinical and radiological findings may be overlapping and molecular studies may be needed for the differential diagnosis of this disorder, such as Gsa bioactivity in patients with AHO. Although AHO patients have low or normal serum calcium levels, patients with acrodysostosis have normal calcium levels. ${ }^{6,7,9}$

In the case herein reported, there was no mental 
retardation and the patient's IQ was normal. Severe craniofacial abnormalities including maxillary hypoplasia, low nasal bridge and triangular mouth were observed in this patient, while skeletal abnormalities such as peripheral dysostosis were also present. No hearing abnormality was detectable. Repeated serum and alkaline phosphatase were found to be elevated while serum PTH levels were low. Though thorough investigations to determine differential diagnosis for hypercalcemia, including endocrine and nonendocrine etiologies, were performed in this patient, no cause for the hypercalcemia was identified. Wilson at al. reported two unrelated patients with a clinical diagnosis of acrodysostosis and they measured Gs $\alpha$ bioactivity in both of them. There was no reduction in the bioactivity of the patients. Mutation analysis of the Gs $\alpha$ gene showed no sequence variation in exons. Serum calcium levels were in the normal range in the patients. On the basis of these findings, they concluded that, at least in a proportion of patients with acrodysostosis, the condition is etiologically distinct from AHO. ${ }^{6}$

Graham et al investigated radiographic findings and Gsa bioactivity in patients with acrodysostosis. In the radiological evaluation they found varying degrees of spinal stenosis in the patients and these finding were accepted as characteristic of acrodysostosis. In Gsalpha bioactivity studies they showed that the patients with acrodysostosis have normal bioactivity of the alpha subunit of the Gs protein, therefore indicating that acrodysostosis has a different pathogenesis from AHO or pseudohypoparathyroidism. All the patients had normal serum calcium levels. ${ }^{7}$ The present study suggest that patients with pseudo-hypoparathyroidism and acrodysostosis may be phenotypically very similar and their radiological findings identical. In these circumstances, molecular analysis such as Gs-alpha bioactivity studies, mutation screening and other radiological findings, such as spinal stenosis, may be needed for differential diagnosis of the disease. For the differential diagnosis of hypercalcemia, the patient was also investigated for other potential causes (primary hyperparathyroidism, malignancy, calcitriol mediated hypercalcemia, benign disorders associated with PTHrP mediated hypercalcemia including HIV or Systemic lupus erythematosus, drug use and psudohypercalcemia) in terms of clinical and laboratory findings. Unfortunately, however, the etiology was not apparent. In conclusion, this case report may be of assistance for the differential diagnosis between AHO and acrodysostosis. Acrodysostosis may be associated with hypercalcemia. The cause of hypercalcemia remains to be determined.

\section{REFERENCES}

1. Jones KL, 1992 Acrodysostosis. In: Jones KL (ed) Lippincott Williams \& Wilkins Press, Philadelphia; pp, 463-464.

2. Robinow M, Pfeiffer RA, Gorlin RJ, et al, 1971 Acrodysostosis: A syndrome of peripheral dysostosis, nasal hypoplasia, and mental retardation. Am J Dis Child 121: 195-203.

3. Butler MG, Rames LJ, Wadlington WB, 1988 Acrodysostosis: Report of a 13-year old boy with review of literature and metacarpophalangieal pattern profile analysis. Am J Med Genet 30: 971-980.

4. Hamanishi C, Nagata Y, Nagao Y, Sohen S, Tanaka S, 1993 Acrodysostosis associated with spinal canal stenosis. Spine 18: 1922-1925.

5. Hernandez RM, Miranda A, Alfaro SK, 1991 Acrodysostosis in two generations: an autosomal dominant syndrome. Clin Genet 39: 376-382.

6. Wilson LC, Oude Luttikhuis MEM, Baraitser M, Kingston HM, Trembath RC, 1997 Normal erythrocyte membrane Gs $\alpha$ bioactivity in two unrelated patients with acrodysostosis. J Med Genet 34: 133-136.

7. Graham JM, Krakow D, Smith AK, Lachman RS, 2001 Radiographic findings and Gs-alpha bioactivity stadies and mutation screening in acrodysostosis indicate a different etiology from pseudohypoparathyroidism. Pediatr Radiol 31: 2-9.

8. Halal F, Dop CV, Lord J, 1985 Differential diagnosis in young women with oligomenorrhea and the pseudopseuduhypoparathyroidism variant of Albright's Hereditary Osteodystrophy. Am J Med Genet 21: 551-568.

9. Bringhurst FR, Demay MB, Kronenberg HM, 1998 Hormones and disorders of mineral metabolism. In: Wilson TD, Foster DW, Kronenberg HM, Larsen PR, (eds.) Williams Textbook of Endocrinology. WB Saunders, Philadelphia; pp,1342-1345.

10. Viljoen D, Beighton B, 1991 Brief clinical report epiphyseal stippling in acrodysostosis. Am J Genet 38: 43-45.

11. Macnicol MF, Makris D, 1988 Acrodysostosis and protrusio acetabuli. J Bone Joint Surg 70-B: 38-39.

12. Abrahamov A, Elstein D, Zimran A, 2000 Type IIIc Gaucher disease and acrodysostosis. Isr Med Assoc J 2: 182.

13. Gupte GL, Kher AS, Kanade SP, Bharucha BA, Sagade $\mathrm{SN}, 1994$ Acrodysostosis with 5 alpha reductase deficiency: an unusual association. Indian J Pediatr 61: 287-290. 